\title{
Enfermidades digitais em vacas de aptidão leiteira: associação com mastite clínica, metrites e aspectos epidemiológicos ${ }^{1}$
}

\author{
Luiz Antônio Franco da Silva ${ }^{2 *}$, Maria Clorinda Soares Fioravanti ${ }^{2}$, Bruno \\ Rodrigues Trindade ${ }^{3}$, Olízio Claudino da Silva ${ }^{2}$, Duvaldo Eurides ${ }^{4}$, Paulo Henrique \\ Jorge da Cunha ${ }^{2}$, Leonardo Marçal da Silva ${ }^{5}$ e Maria Ivete de Moura ${ }^{6}$
}

\begin{abstract}
Silva L.A.F., Fioravanti M.C.S., Trindade B.R., Silva O.C., Eurides D., Cunha P.H.J., Silva M.L. \& Moura M.I. 2004.Foot diseases in dairy cows: association with clinical mastitis, metrites and predisposed factors.] Enfermidades digitais em vacas de aptidão leiteira: associação com mastite clínica, metrites e aspectos epidemiológicos. Pesquisa Veterinária Brasileira 24(4):217-222. Escola de Veterinária, Universidade Federal de Goiás, Cx. Postal 131, Goiânia, GO 74001-970, Brazil. E-mail: lafranco@vet.ufg.br

With the objective to investigate a possible association between foot diseases, clinical mastitis and/or metritis and predisposing factors for foot diseases, 5300 dairy cows from 80 intensive and semi-intensive farms were used. In 325 (6.13\%) cows only foot disease was diagnosed, in 35 (0.66\%) foot disease and clinical mastitis, in $52(0.98 \%)$ foot disease and metritis, in $28(0.53 \%)$ foot disease, clinical mastitis and metritis, in $128(2.42 \%)$ only metritis, in $165(3.11 \%)$ only clinical mastitis, and in $89(1.68 \%)$ cows metritis and clinical mastitis. Rapid changes in the diet, high exposure time of hoof horn to slurry and wet conditions, concrete floors, use of footbaths, low frequency of claw trimming, irregular quarantine, and no attention to health aspects during acquisition of the animals were considered as major risk factors for the occurrence of those diseases. The McNemar test for dependent samples showed significant statistical differences between the occurrence of foot disease, clinical mastitis and metritis, in spite of the poor association between those diseases. It was concluded that there was no expressive relationship between foot diseases, clinical mastitis and metritis in lactent cows.
\end{abstract}

INDEX TERMS: Foot diseases, mastitis, metritis, epidemiological factors.

RESUMO.- Utilizaram-se nesse estudo 5300 vacas de aptidão leiteira, provenientes de 80 propriedades rurais, que adotavam manejo intensivo ou semi-extensivo, com o objetivo de averiguar a existência de possível associação entre enfermidades digitais, mastite clínica e/ou metrite e identificar possíveis fatores

\footnotetext{
${ }^{1}$ Recebido em 20 de junho de 2003.

Aceito para publicação em 26 de julho de 2004.

2 Depto Medicina Veterinária, Escola de Veterinária, Universidade Federal de Goiás (UFG), Cx. Postal 131, Goiânia, GO 74001-970. *Autor para correspondência. E-mail: lafranco@vet.ufg.br

${ }^{3}$ Programa de Pós-graduação em Medicina Veterinária, Escola de Vete-

${ }^{4}$ Escola de Veterinária, Universidade Federal de Uberlândia.

${ }^{5}$ Médico Veterinário, Empresa Classivet, Orizona, GO.

${ }^{6}$ Curso de Medicina Veterinária, Centro de Ciências Agrárias, Campus Avançado de Jataí da UFG.
} rinária, UFG. de risco das enfermidades digitais. Em 325 (6,13\%) vacas foram diagnosticados apenas enfermidades digitais, em $35(0,66 \%)$ enfermidades digitais e mastite clínica, em $52(0,98 \%)$ enfermidades digitais e metrite, em $28(0,53 \%)$ enfermidades digitais, mastite clínica e metrite, em $128(2,42 \%)$ apenas metrite, em $165(3,11 \%)$ somente mastite clínica, e em $89(1,68 \%)$ vacas metrite e mastite clínica. As mudanças bruscas na alimentação, o excesso de sujidades nas instalações, os pisos irregulares e abrasivos, a não utilização ou uso incorreto de pedilúvio, a falta de casqueamento preventivo, a ausência de quarentena, e a aquisição de animais sem a preocupação com o aspecto sanitário foram considerados os fatores de risco de maior ocorrência. Foi constatada diferença significativa entre a ocorrência de enfermidades digitais, mastite clínica e metrite, além de associação fraca entre tais enfermidades, concluindo-se que não houve relação expressiva entre enfermidades podais, mastite clínica e metrite em vacas lactantes. 
TERMOS DE INDEXAÇÃO: Enfermidades digitais, mastite, metrite, fatores epidemiológicos.

\section{INTRODUÇÃO}

As enfermidades digitais dos bovinos apresentam impacto econômico negativo sobre a rentabilidade da pecuária mundial, tanto pela redução da produtividade quanto pelo aumento nos custos dos tratamentos, bem como o descarte prematuro de animais de alto valor zootécnico (Corbellini 1994).

Avaliando os parâmetros reprodutivos em vacas leiteiras Ramos et al. (2001) relataram que a taxa de descarte de animais com enfermidades digitais foi de $25 \%$ e Silva (2002) confirmou a importância econômica das doenças de casco destacando os seus efeitos sobre a produção, a menor eficiência reprodutiva dos touros, o aumento no período de serviço e os altos custos dos tratamentos. Acrescentou que as doenças dos cascos podem ser um importante fator limitante na manutenção de bons níveis de fertilidade, mas a associação dessas enfermidades com a redução dos níveis de fertilidade possui diversas questões inexplicáveis.

No Brasil, as doenças digitais dos bovinos só perdem em prejuízos econômicos para mastites e para as patologias da reprodução, uma vez que têm afetado em média de 11 a $25 \%$ das vacas leiteiras (Dias 1996). Em um trabalho realizado na Eslovênia por Orenisk (1995), utilizando vacas leiteiras Friesian, verificouse $15,1 \%$ de enfermidades digitais, sendo que algumas vacas apresentavam desordens reprodutivas associadas com essas patologias.

Segundo Grunert \& Berchtold (1995), a retenção de anexos fetais em bovinos acarretou o estabelecimento de metrites puerperais. Nilsson (1963) relatou que a histamina produzida em úteros potadores de metrites e de retenção de anexos fetais, podia desencadear laminite. Para Boosman et al. (1991), as endotoxinas produzidas nesses casos estão relacionadas com a etiopatogenia das enfermidades podais.

Allenstein (1981), Edwards (1982) e Liversey \& Fleming (1984), apontaram as substâncias vasoativas, histamina e endotoxinas, produzidas em decorrência das doenças sistêmicas no período pós-parto tais como acetonemia, mastite, metrite e acidose rumenal, como responsáveis por danos ao córion laminar. Chamaram a atenção para o fato da laminite aguda ter sido observada em animais portadores de doenças em que essa modalidade de endotoxina podia estar presente. Em estudos conduzidos por Vermunt \& Greenough (1994), foi verificada a ocorrência de laminites em bovinos submetidos a altas doses de endotoxinas administradas via artérias digitais. Silva (2002) acrescentou que o excesso de grãos, milho e rações essencialmente protéicas, além das enfermidades da glândula mamária e do útero, são fatores de risco da laminite.

Peeler et al. (1994) constataram significativa associação entre enfermidades dos cascos e mastite no período anterior à estação de monta. Os autores acreditaram que fatores relacionados ao manejo poderiam predispor o animal a ambas as condições. Ressaltaram que as distocias fetais eram fatores significativos para o surgimento de mastites e doenças podais antes do serviço de cobertura. Deduziram ainda que os traumatismos durante o parto aumentavam a susceptibilidade às doenças comuns ao período pós-parto.
Este trabalho teve como objetivo verificar possíveis associações entre enfermidades digitais, metrite e mastite clínica em vacas de aptidão leiteira no período pós-parto, criadas em regime intensivo ou semi-extensivo, e avaliar possíveis fatores de risco envolvidos na etiopatogenia das enfermidades digitais.

\section{MATERIAL E MÉTODOS}

No período compreendido entre janeiro de 1996 a março de 2002, foram examinadas 5.300 fêmeas bovinas de aptidão leiteira, procedentes de propriedades rurais do Estado de Goiás, que adotavam os manejos intensivo ou semi-extensivo. Quando os bovinos eram mantidos confinados a alimentação básica constituía-se de silagem de milho enriquecida com concentrado e quando o confinamento ocorria apenas em um determinado período, os animais eram suplementados com silagem de milho, sorgo, concentrado ou canade-açúcar associada à uréia. Nesses criatórios, durante a estação das chuvas, os animais eram mantidos em regime de pastagem com gramíneas do gênero Brachiaria sp ou Andropogon gayanus.

Todos os bovinos foram examinados segundo Rosenberg (1989) com a finalidade de estabelecer uma possível relação entre a presença de enfermidades digitais, mastite clínica ou metrite, mas sem considerar nessa fase do estudo se a presença de uma seria em consequiência da outra. Do total de animais examinados, 822 fêmeas estavam em lactação e eram portadoras de no mínimo uma dessas enfermidades. A classificação das doenças digitais foi realizada, segundo Greenough (1994) e Silva et al. (1997). O diagnóstico de mastite clínica fundamentouse nos sinais de hiperemia, dor, calor e endurecimento da glândula mamária, descritos por Ladeira (2001). Acrescentaram-se ainda alterações no leite como a presença de grumos e/ou sangue identificados pelo uso da caneca de fundo preto e/ou California Mastitis Test (CMT) (Schalm \& Noorlander 1957). Para avaliação das fêmeas portadoras de metrite utilizou-se o exame ginecológico realizado por meio da palpação retal, o uso de espéculo vaginal, e ainda fundamentou-se no histórico e nos sinais clínicos de anestro, descargas vaginais e retenção de envoltórios fetais (Rebhun 2000).

Após criterioso exame clínico procedeu-se a distribuição dos animais em sete grupos, assim constituídos: Grupo 1 (I) fêmeas portadoras de doenças digitais; Grupo 2 (II) bovinos acometidos de enfermidades podais e mastite clínica; Grupo 3 (III) animais com doenças de casco e metrite; Grupo 4 (IV) bovinos portadores de doenças digitais, concomitante com mastite clínica e metrite; Grupo 5 (V) animais que apresentavam apenas metrite; Grupo 6 (VI) bovinos portadores exclusivamente de mastite clínica; Grupo 7 (VII) vacas com diagnóstico positivo de metrite e mastite clínica.

Paralelamente, preencheu-se um questionário contendo indagações sobre possíveis fatores de risco que pudessem estar envolvidos na etiopatogenia das enfermidades digitais diagnosticadas, tais como alimentação, higiene das instalações, estação do ano, quarentena, uso do pedilúvio, casqueamento, aspectos sanitários entre outros.

$\mathrm{Na}$ análise estatística utilizou-se a prova de McNemar para a comparação entre duas proporções com amostras dependentes (Curi 1997).

\section{RESULTADOS E DISCUSSÃO}

De um universo de 5300 animais avaliados, 822 apresentaram enfermidades digitais, ou mastite clínica ou metrite, associadas ou não. Desse total, 325 (39,54\%), eram portadores apenas de doenças podais, $35(4,26 \%)$ apresentavam enfermidades digitais e mastite clínica, em 52 (6,32\%) foram diagnosticadas doenças 
Quadro 1. Distribuição em grupos, de fêmeas bovinas de aptidão leiteira, criadas em regime intensivo ou semi-extensivo, portadoras de enfermidades digitais, associadas ou não a mastite clínica ou metrite, no período de janeiro de 1996 a março de 2002

\begin{tabular}{|c|c|c|c|c|c|c|c|c|c|c|c|c|c|c|}
\hline \multirow[t]{2}{*}{ Grupos $^{\mathrm{a}}$} & \multicolumn{13}{|c|}{ Enfermidades $^{\mathrm{b}}$} & \multirow[t]{2}{*}{ Total } \\
\hline & LAM & PIV & PNC & DVR & DDG & FIT & SLD & DLB & FIC & ERT & ABS & ULS & DEU & \\
\hline I & 9 & 28 & 124 & 3 & 106 & 13 & 8 & 5 & 4 & 4 & 3 & 7 & 11 & 325 \\
\hline II & 3 & 1 & 6 & - & 18 & - & 1 & - & 1 & 2 & - & 1 & 2 & 35 \\
\hline III & 2 & 3 & 10 & 1 & 29 & 1 & - & 1 & - & 1 & 1 & 1 & 2 & 52 \\
\hline IV & 2 & 1 & 5 & - & 9 & 1 & 2 & - & 2 & 4 & - & 1 & 1 & 28 \\
\hline Total & 16 & 33 & 145 & 4 & 162 & 15 & 11 & 6 & 7 & 11 & 4 & 10 & 16 & 440 \\
\hline
\end{tabular}

${ }^{\mathrm{a}} \mathrm{GI}=$ Animais com enfermidades digitais; GII = animais portadores de enfermidades podais e mastite clínica; GIII = bovinos com doenças digitais e metrite clínica; GIV = fêmeas bovinas com enfermidades digitais, mastite clínica e metrite.

${ }^{\mathrm{b}} \mathrm{LAM}=$ laminite; PIV = pododermatite interdigital vegetativa; PNC = pododermatite necrosante; DVR = dermatite verrucosa; DDG = dermatite digital; FIT = flegmão intergital; SLD = sola dupla; DLB = doença da linha branca; FIC = fissura do casco; ERT = erosão do talão; ABS = abscesso solear; ULS = úlcera da sola; DEU = deformação ungular.

dos cascos e metrite e em 28 (3,41\%), além das enfermidades podais, identificou-se a presença de mastite clínica e metrite, totalizando-se $440(53,53 \%)$ bovinos (Quadro 1$)$.

Dentre os $382(46,47 \%)$ bovinos que não eram portadores de enfermidades digitais, em $128(15,57 \%)$ foi diagnosticada metrite, em $165(20,07 \%)$ mastite clínica e em $89(10,83 \%)$ metrite e mastite clínica, correspondendo, respectivamente, aos grupos V, VI e VII (Figura1).

Das 80 propriedades rurais atendidas, $25(31,25 \%)$ adotavam o manejo intensivo e em $55(68,75 \%)$ o sistema era semi-extensivo. Independente do manejo e dos grupos nos quais as 440 fêmeas foram alocadas, as enfermidades de casco de maior ocorrência foram a dermatite digital (DDG) com 162 casos $(36,82 \%)$, seguida da pododermatite necrosante (PNC) com 145 bovinos enfermos (32,95\%) e da pododermatite interdigital vegetativa (PIV) com 33 bovinos, representando 7,50\% do total de enfermi-

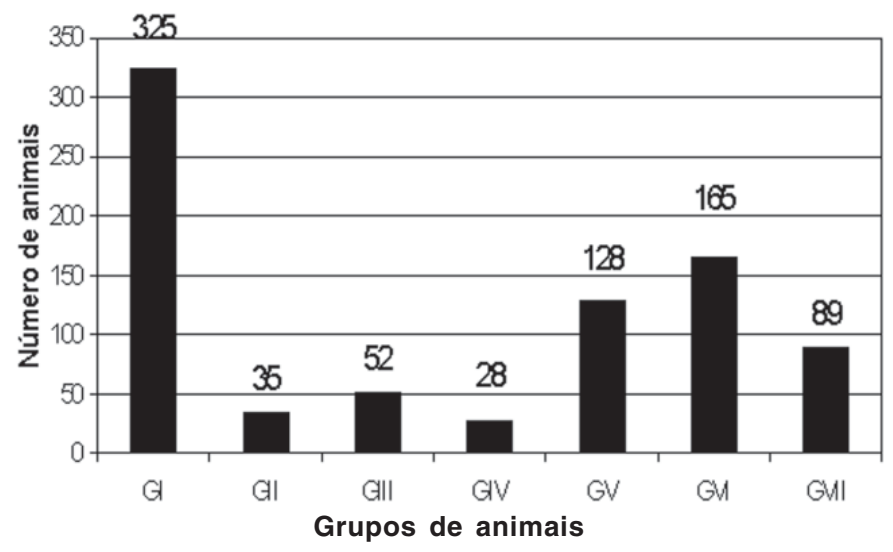

$\mathrm{GI}=$ animais com enfermidades digitais; GII = animais portadores de enfermidades podais e mastite clínica; GIII = bovinos com doenças digitais e metrite clínica; GIV = fêmeas bovinas com enfermidades digitais, mastite clínica e metrite; GV = animais que apresentavam apenas metrite; GVI = bovinos portadores exclusivamente de mastite clínica; GVII = vacas com diagnóstico positivo de metrite e mastite clínica.

Fig. 1. Fêmeas bovinas em lactação portadoras de diferentes enfermidades digitais, metrite e mastite clínica associadas ou não distribuídas nos respectivos grupos, no período de janeiro de 1996 a março de 2002.
Quadro 2. Fêmeas bovinas de aptidão leiteira, criadas em regime intensivo ou semi-extensivo, portadoras de enfermidades digitais, no período de janeiro de 1996 a março de 2002

\begin{tabular}{lc}
\multicolumn{1}{c}{ Enfermidades } & Total \\
\hline Laminite & 16 \\
Pododermatite interdigital vegetativa & 33 \\
Pododermatite necrosante & 145 \\
Dermatite verrucosa & 4 \\
Dermatite digital & 162 \\
Flegmão intergital & 15 \\
Sola dupla & 11 \\
Doença da linha branca & 6 \\
Fissura do casco & 7 \\
Erosão do talão & 11 \\
Abscesso solear & 4 \\
Úlcera da sola & 10 \\
Deformação ungular & 16 \\
Total & 440
\end{tabular}

dades podais diagnosticadas (Quadro 2).

Molina et al. (1999) em um estudo realizado com 469 vacas no período de lactação e criadas em regime de confinamento, em criatórios da bacia leiteira de Belo Horizonte, MG, encontraram lesões em 142 animais $(30,3 \%)$ sendo as mais freqüentes a erosão da camada córnea com $48,5 \%$, seguida pela dermatite interdigital com $13,5 \%$ e da pododermatite séptica com 9,6\% do total de animais avaliados. Marega (2001) avaliando a ocorrência de dermatite digital em bovinos concluiu que o tipo de exploração não exerce influência sobre a prevalência, sendo que a enfermidade pode ser encontrada tanto em rebanhos de corte quanto de leite. $\mathrm{O}$ autor não mencionou a associação entre doenças digitais, mastite e metrite, porém atentou para o fato de que a utilização de pedilúvio e casqueamento dos animais, a lotação e a presença de solos úmidos e sujos são fatores de risco importantes na disseminação da doença.

Foi constatada diferença significativa $(p<0,05)$ entre a freqüência de ocorrência de distúrbios digitais e metrite, na amostra estudada e o coeficiente de associação indicou associação fraca entre as duas enfermidades. Os mesmos resultados foram encontrados quando se avaliou a relação entre enfermidades podais e mastite clínica. Para Singh et al. (1993), as doenças 
digitais têm etiologia multifatorial envolvendo fatores como a genética, nutrição, meio ambiente, tipo de piso, idade, parição, estação do ano e clima. De acordo com Ramos (1999), a higiene precária das instalações, o excesso de umidade, a utilização de cascalho nas vias de acesso ou dentro dos estábulos, e a presença de irregularidades nos pisos, bem como as mudanças bruscas na alimentação e o fornecimento de volumosos com qualidade questionável, foram considerados possíveis fatores predisponentes para o desenvolvimento das lesões de casco. Como agravante foi citada a não adoção de medidas preventivas como toalete de casco e a utilização de pedilúvio. Segundo Peeler et al. (1994), existe uma associação entre doenças digitais e mastite, mas não citaram o grau de associação entre as duas enfermidades.

Dentre os 317 casos diagnosticados de mastite clínica, apenas $63(19,87 \%)$ apresentavam, concomitantemente, doenças digitais. Calavas et al. (1996) estudando a recorrência de mastite clínica em vacas, entre lactações, observaram uma maior tendência de ocorrência desse fenômeno entre o primeiro e o quinto dia pós-parto, mas os autores não se preocuparam em estudar a possível relação entre doenças digitais e da glândula mamária. A possibilidade de recorrência da mastite clínica foi também apontada por Cobo-Abreu et al. (1979), Bunch et al. (1984) e Dohoo \& Martin (1984). Syvajarvi et al. (1986) relacionaram a recorrência de mastite a um componente genético que, segundo Nickerson (1985) o processo poderia ser explicado pela dificuldade de mobilização da imunidade local nos primeiros estágios da lactação. Por esse motivo não estaria ligada a determinados fatores de risco como as enfermidades digitais.

Admite-se que, além da possibilidade da mastite clínica e da metrite estarem envolvidas na gênese das enfermidades digitais dos bovinos utilizados neste estudo, existem indícios de que fatores relacionados ao manejo nos diferentes criatórios também tenham influenciado no aparecimento das doenças avaliadas. Acrescente-se como justificativa o fato de que em propriedades, nas quais o manejo era intensivo, os animais chegavam a permanecer até 16 horas diariamente em posição quadrupedal para serem ordenhados ou para se alimentarem. Essa observação encontra respaldo nas afirmações de Greenough (1997), o qual afirmou que a intensificação da produção compromete o bem estar animal, interfere na fisiologia do sistema digestivo e conseqüentemente, contribui para o aumento na ocorrência de enfermidades digitais em bovinos leiteiros. Segundo Silva (2002), algumas propriedades rurais têm praticado até três ordenhas diárias, deixando pouco tempo para o animal descansar. Para responder em produção, o bovino necessita passar boa parte do tempo em posição quadrupedal para se alimentar, além de permanecer, na maioria das vezes, em pisos de concreto abrasivo e irregular.

Do total de bovinos diagnosticados com mastite clínica, $117(36,91 \%)$ apresentavam metrite associada. Mota et al. (1999) estudaram um foco de mastite clínica e subclínica em bovinos causado pela Candida sp e identificaram que o ordenhador era a fonte de infecção, uma vez que os animais não apresentavam outras manifestações clínicas tais como metrites. Os sinais clínicos restringiam-se aos úberes, os quais se apresentavam edemaciados, com aumento de sensibilidade, hiperêmicos, com grumos na secreção láctea, pus e estrias de sangue.

Ficou evidente, após criteriosa anamnese, que outros fatores de risco além da metrite e da mastite clínica, tais como mudanças bruscas na alimentação, excesso de sujidades nas instalações, a falta de critério na aquisição e introdução dos animais nas propriedades, os pisos irregulares e abrasivos, a promiscuidade entre bovinos doentes e sadios a ausência de quarentena, o uso incorreto ou a não utilização do pedilúvio e a ausência de casqueamento preventivo, também podem estar relacionados com a etiopatogenia das diferentes doenças digitais diagnosticadas nos bovinos deste estudo. Segundo Silva (1998), vários fatores podem predispor a enfermidades dos cascos, entre eles algumas doenças do aparelho reprodutor e da glândula mamária, o excesso de umidade, os terrenos duros e irregulares, a raça, a idade, a época de parição, doenças imunossupressoras, carência de macro e micro minerais, indigestão e traumatismos. Nilsson (1963) e Boosman et al. (1991) indicaram a metrite como um dos fatores desencadeantes da laminite. Dohoo \& Martin (1984), Faye et al. (1986), Rowlands et al. (1986) e Gröhn et al. (1990) mencionaram a associação entre retenção de envoltórios fetais e metrite. Segundo Toussain-Raven (1992), a retenção de envoltórios fetais produziria uma hiperhistaminemia responsável pela laminite.

Considerando-se os casos diagnosticados de mastite clínica e a sua possível relação com enfermidades digitais, pode ser adotado raciocínio similar ao anterior. Para Allenstein (1981), existiam fortes indícios de que as mastites possam estar relacionadas com a ocorrência de laminites. Todavia, após avaliar criteriosamente o pequeno número de casos de laminite clínica em relação às outras doenças digitais observadas nos bovinos desse estudo é possível inferir que esta patologia mamária tenha, inicialmente, mesmo quando sua manifestação fora subclínica, desencadeado um quadro de toxemia e/ou septicemia e por via hematógena essas substâncias teriam atingido as extremidades dos membros locomotores, provocando microlesões no endotélio vascular, liberação de histamina seguida de vasodilatação que, em tese, teria comprometido o cório laminar e consequentemente, a qualidade do estojo córneo. Para Mendonça (2001), bovinos portadores de cascos com a qualidade comprometida, seriam mais susceptíveis às enfermidades podais. Segundo Rebhum (2000), as mastites, principalmente, as ambientais, causadas por coliformes fecais (Gram negativos), produzem endotoxinas que causam no úbere complicações locais, como inchaço, agalactasia e complicações sistêmicas, distribuídas entre lesões músculo-esqueléticas secundárias, distúrbios metabólicos e laminite. Allenstein (1981), Liversey \& Fleming (1984), Orenisk (1995) e Grunert \& Berchtold (1995) limitaramse a sugerir a existência de relação entre metrites, mastites e enfermidades digitais, principalmente pela produção de endotoxinas, mas sugeriram a participação de outros fatores de risco.

Dentre os 440 animais portadores de enfermidades podais, somente $80(18,18 \%)$ apresentavam também metrite. Calavas et al. (1996) encontraram uma relação negativa entre mastite clínica e metrite após o sexto dia da parição durante a lactação se- 
guinte. Mastites e metrite compartilham o mesmo componente não específico infeccioso que, segundo Dubois et al. (1980) e Mascarenhas (1987), pode resultar no mesmo tipo de imunidade. Em parte, poderia atribuir esse achado, à estimulação da disponibilidade de leucócitos, bem como à mobilidade dessas células, resultante da mastite, podendo promover melhor imunidade durante a lactação seguinte.

Dentre os animais selecionados para este trabalho 616, (75\%) encontravam-se nos primeiros meses de lactação. É muito provável que nesse período, além de estar sobre o efeito do estresse pós-parto, a fêmea geralmente depara-se com distúrbios metabólicos decorrentes de falhas no metabolismo energético em função da maior exigência nutricional necessária à mantença da produção de leite, tanto em qualidade como em quantidade. Um outro achado constante nos diferentes criatórios e que pode ser considerado como fator de risco nas propriedades aqui estudadas, era a exposição dos animais a uma dieta rica em carboidratos facilmente fermentáveis e concentrados protéicos sem uma prévia adaptação da microbiota rumenal. Sabidamente, essa prática pode causar alterações sistêmicas e conseqüientemente, uma laminite. Essa possibilidade também foi defendida por Rebhun (2000), o qual observou que vacas não adaptadas a dietas próprias para lactação e ricas em energia, juntamente com a liberação de endotoxinas ou de outros mediadores a partir de infecções como metrite e mastites sépticas, ocasionalmente podem contribuir para a etiopatogenia da laminite. Segundo Nocek (1993), as laminites ou outras enfermidades podais são mais comuns nos períodos próximos à parição e nos 2 a 3 meses iniciais da lactação. Para Clarkson et al. (1996), as lesões de casco representam 79\% dos distúrbios locomotores dos bovinos, e Offer et al. (2000) afirmaram que a patogenia das doenças digitais provavelmente seja uma das menos compreendidas. Estudos realizados por Leonard et al. (1995) e Leach et al. (1997) demonstraram que os distúrbios dos membros locomotores podiam ser influenciados por muitos fatores, tais como, alojamento, estação do ano, nutrição, conformação do casco e genótipo. Chaplin et al. (1999) apontaram ainda como possíveis fatores de risco das doenças digitais, mudança de comportamento e interação social dos animais.

Apesar de todas as vacas utilizadas neste estudo encontrarem-se no período pós-parto e, mesmo seguindo as considerações de Rebhum (2000), não foi possível estabelecer as causas das metrites encontradas em $80(26,94 \%)$ bovinos distribuídos nos grupos III, IV, V e VII. Entretanto, acredita-se que o estresse decorrente do parto, as mudanças hormonais e a retenção dos envoltórios fetais diagnosticados em $17(21,25 \%)$ podem ter tornado as fêmeas mais vulneráveis a infecções uterinas. Acrescenta-se ainda que a operação cesariana realizada em $3(3,75 \%)$ animais e a extração forçada praticada em $5(6,25 \%)$, com a finalidade de solucionar partos distócicos, podem também ter contribuído para o desencadeamento das metrites diagnosticadas nos bovinos desta pesquisa. Segundo Rebhum (2000), a metrite pósparto inicial ocorre em 93,00\% dos bovinos leiteiros e a cura geralmente aconteceu em aproximadamente 40 dias após o nascimento do bezerro, mas não atribuiu a presença de doenças digitais como conseqüência de enfermidades uterinas.

\section{CONCLUSÕES}

Existe associação fraca entre enfermidades digitais, mastite clínica e metrite em vacas de aptidão leiteira oriundas de criatórios intensivos e semi-extensivos.

As metrites e as mastites clínicas não puderam ser consideradas, isoladamente, como responsáveis pelas doenças digitais diagnosticadas, uma vez que vários fatores de risco podem estar envolvidos na etiopatogenia dessas enfermidades.

\section{REFERÊNCIAS}

Allenstein L.C. 1981. Lameness of cattle. Can.Vet. J. 22:65-67.

Boosman R., Nemeth F. \& Gruys F. 1991. Bovine Laminitis: clinical aspects, pathology and pathogeneses with reference to acute bovine laminitis. Vet. Rec.125:573-575.

Bunch K.J., Henegan D.J.S., Hilit K.G. \& Rowlands G.J. 1984. Genetic influences on clinical mastitis and its relationships with milk yield, season and stage of lactation. Livest. Prod. Sci.11:91-104.

Calavas D., Faye B., Bugnard F., Ducrot C. \& Raymond F.1996. Analysis of associations among diseases in French dairy cows in two consecutive lactations. Prev. Vet. Med. 27(1):43-55.

Chaplin S.J., Ternent H.E., Offer J.E., Logue D.N. \& Knight C.H. 1999. A comparison of hoof lesions and behaviour in pregnant and early lactation heifers at housing. Vet. J. 159:147-153.

Clarkson M.J., Downham D.Y., Faul W.B., Hughes J.W., Manson F.J., Merrit J.B., Murray, R.D., Russell W.B., Sutherst, J. \& Ward W.R. 1996. Incidence and prevalence of lameness in dairy cattle. Vet. Rec. 138:563-567.

Cobo-Abreu R., Martin S.W., Stone J.B. \& Willoughby R.A. 1979. The rates of pattern of survivorships and disease in a university dairy herd. Can. Vet. J. 20:177-183.

Corbellini C.N. 1994. Fatores nutricionales relacionados com las afecciones podales en bovinos. Enf. Pod. Bov., Santiago, Chile. 32p.

Curi P.R. 1997. Metodologia e análise da pesquisa em ciências biológicas. Editora Roca, São Paulo. 263p.

Dias R. S. 1996. Tratamento de cascos se faz com informações e critérios. Revta Balde Branco 385(3):26-29.

Dohoo I.R. \& Martin S.W. 1984. Disease, production and culling in HolsteinFriesian cows. III. Disease and production as determinants of disease. Prev. Vet. Med. 2:671-690.

Dubois J.A., Wordinger R.J. \& Dickey J.F. 1980. Tissue concentrations of mast cells and lymphocytes of the bovine uterine tube (oviduct) during the oestrous cycle. Am. J. Vet. Res. 41:806-808.

Edwards G.B. 1982. Acute and subacute laminitis in cattle. Vet. Annu. 22:99-106.

Faye B. \& Brochart M. 1986. Enquête éco-pathologique continue. Approche épidémiologique des mammites post-partum chez la vache laitière: étude descriptive et typologic des élevages. Ann. Rech. Vét.17:297-311.

Greenough P.R.1994. Structure and function of the digit. Proc. VIII Int. Conf. Bovine Lamness, Banff, Holland, p.82-91. (Abstract)

Greenough P.R. 1997. Cascos irregulares são sinais de problemas. Revta Balde Branco 388(2):9-13.

Gröhn Y.T., Mc Culloch C.E. \& Saloniemi, H.S. 1990. Epidemiology of reproductive disorders in dairy cattle: associations among host characteristics disease and production. Prev. Vet. Med. 8:25-39.

Grunert E. \& Berchtold M. 1995. Fertilitätsstörungen beim weiblichen Rind. Parey Verlag, Berlin. 315p.

Ladeira, S.R.L. 2001. Mastite bovina, p.294-307. In: Riet-Correa F., Schild A.L., Méndez M.D.C. \& Lemos R.A.A. (ed.) Doenças dos Ruminantes e dos Eqüinos. Vol. 2. Editora Varela, São Paulo.

Leach K.A., Logue D.N., Kempson S.A., Offer J.E., Ternent H.E. \& Randall J.M. 1997. Claw horn lesions in dairy cattle: development of sole and white line haemorrhages during the first lactation. Vet. J. 154:215-225. 
Leonard F.C., O'connell J. \& O'farrell K. 1995. Effect of overcrowding on claw health in first-calved Friesian heifers. Brit. Vet. J. 152:459-472.

Liversey C.T. \& Fleming F.L. 1984. Nutricional influencis on laminitis, sole ulcer and bruised sale in Friesian cows. Vet. Rec. 114:510-512.

Marega L.M. 2001. Ocorrência e tratamento de lesões podais semelhantes a dermatite digital em bovinos. Dissertação de mestrado, Universidade Estadual Paulista, Jaboticabal, São Paulo. 72p.

Mascarenhas R. 1987. Les endométrites non spécifiques de la vache. I. Les sécrétions utérines et la réaction inflammatoire de l'endomètre. II. Les indicateurs de l'inflammation dans les sécrétions cervico-vaginales: relation avec la feritilité. Thèse Doct., Univ. Clermont, Clemont-Ferrand, France. 236p.

Medonça A.C. 2001. Aspectos morfológicos e morfométricos dos dígitos de bovinos das raças gir e holandesa. Dissertação de mestrado, Universidade Federal de Goiás, Goiânia. 57p.

Molina L.R., Carvalho A.U., Facury Filho E.J., Ferreira P.M. \& Ferreira V.C.P. 1999. Prevalência e classificação das afeç̧ões podais em vacas lactantes na bacia leiteira de Belo Horizonte. Arq. Bras. Med. Vet. Zootec. 51(2):149152.

Mota R.A., Costa N.A., Oliveira A.A.F., Sá M.E.P. \& Almeida Neto J.B. 1999. Bovine mastitis caused by Candida $s p .:$ epidemiological and clinical aspects. Revta Bras. Ciênc. Vet. 6(2):101-103.

Nickerson S.C. 1985. Immune mechanisms of the bovine udder: an overview. J. Am. Vet. Med. Assoc. 1:41-45.

Nilsson S.A. 1963. Clinical, morphological and experimental studies of laminitis in cattle. Ac. Vet. 304p.

Nocek J.E. 1993. Hoof Care for dairy cattle. W.D. Heard \& Co., Pennsylvania. $32 \mathrm{p}$.

Offer J.E., Mcnulty D.N. \& Logue, D.N. 2000. Observations of lameness, hoof conformation and developement of lesions in dairy cattle over four lactations. Vet. Rec. 147:105-109.

Orenisk A.1995. Effect of health and reproductive desordens on milk yield and fertility in dairy cows. Bov. Prac. 29:43-44.
Peeler E.J., Otte M.J. \& Esslemont R.J. 1994. Inter-relationships of periparturiente diseases in dairy cows. Vet. Rec. 5:129-132.

Ramos L.S., Silva L.A.F., Meirinhos M.L.G., Juliano R.S., Prado L.F., Cunha P.H.J., Moraes R.R.\& Fioravanti M.C.S. 2001. Avaliação de parâmetros reprodutivos em fêmeas bovinas de aptidão leiteira portadoras de pododermatite necrosante. Ars Vet. 17(2):98-106.

Ramos L.S. 1999. Avaliação econômica dos efeitos da pododermatite sobre a reprodução e produção dos bovinos. Dissertação de mestrado, Universidade Federal de Goiás, Goiânia, Goiás. 113p.

Rebhun W.C. 2000. Doenças do Gado Leiteiro. Editora Roca, São Paulo.140p.

Rosenberger G. 1989. Enfermidades de los Bovinos. Vol 2. Editora Hemisfério Sul, Buenos Aires. 135p.

Rowlands G.J., Lucey S.\& Russel A.M. 1986. Susceptility to disease in the dairy cow and its relationships with occurrences of other diseases in the current or preceding lactation. Prev. Vet. Med. 167:105-111.

Schalm O.W. \& Noorlander P.O. 1957. Experiments and observations leading to development of the California Mastitis Test. J. Am. Vet. Med. Assoc. 130(5): 199-204.

Silva L.A.F., Silva C.A., Fioravanti M.C.S., Araújo E.G., Vieira D. \& Borges, N.C. 1997. Estudo comparativo entre três tratamentos cirúrgicos para duas formas de apresentação clínica da pododermatite bovina, p.34. 24ํㅡㄹ. Congr. Bras. Med. Vet., Goiânia, Brasil.

Silva L.A.F. 1998. Haja casco para tanta doença. Revta Prod. 22(3):17-21.

Silva L.A.F. 2002. Mudanças trazem doenças de casco em Goiás. Revta DBO, São Paulo, 262:156-160.

Singh S.S., Ward W.R. \& Murray, P.D. 1993. Etiology and patogeneses of sole lesions causing lameness in cattle: a review. Vet. Bull. 63(4):7-9.

Syväjärvi J., Saloniemi H. \& Gröhn Y. 1986. A epidemiological and genetic study on registered diseases in Finnish Ayrshire cattle. Acta Vet. Scand. 27:233-234.

Toussain-Raven E. 1992. Cattle Footcare and Claw Trimming. Farming Press United, Ipswich, UK. 125p.

Vermunt J.J. \& Grenough, P. R. 1994. Predisposing factors of laminitis in cattle. Brit.Vet. J. 150(2):150-164. 